\title{
The Mysterious Magnetospheres of Magnetars
}

\section{Matthew G. Baring*}

Department of Physics and Astronomy - MS 108, Rice University, 6100 Main Street, Houston, Texas 77251-1892, USA

E-mail: baringerice.edu

\section{Zorawar Wadiasingh}

Gravitational Astrophysics Laboratory, Code 663, NASA's Goddard Space Flight Center, Greenbelt, Maryland, 20771, USA ${ }^{\dagger}$

E-mail: zwadiasingh@gmail.com

\section{Peter L. Gonthier}

Hope College, Department of Physics, 27 Graves Place, Holland, MI 49423, USA

E-mail: gonthier@hope.edu

\section{Alice K. Harding}

Theoretical Division, Los Alamos National Laboratory, Los Alamos, NM 87545, USA

E-mail: ahardingx@yahoo.com

\section{Kun Hu}

Department of Physics and Astronomy - MS 108, Rice University, 6100 Main Street, Houston, Texas 77251-1892, USA

E-mail: kh38erice.edu

\begin{abstract}
Magnetars are the most luminous compact objects in the stellar mass range observed in the Milky Way, with giant flares of hard X-ray power $\gtrsim 10^{45} \mathrm{erg} / \mathrm{sec}$ being detected from three soft gamma repeaters in the Galactic neighborhood. Periodicity seen in magnetar persistent emission, and a distinctive "spin-down" lengthening of this period, have driven the paradigm that stronglymagnetized neutron stars constitute these fascinating sources. The steady X-ray emission includes both thermal atmospheric components, and magnetospheric contributions that are manifested as hard X-ray "tails." This paper addresses observational and theoretical elements pertinent to the steady hard X-ray emission of magnetars, focusing on dissipative processes in their magnetospheres, and elements of Comptonization and polarization. It also discusses the action and possible signatures of the exotic and fundamental QED mechanisms of photon splitting and magnetic pair creation, and the quest for their observational vindication.
\end{abstract}

7th Annual Conference on High Energy Astrophysics in Southern Africa ***

28 - 30 August 2019

Swakopmund, Namibia

\footnotetext{
*Speaker.

${ }^{\dagger}$ Universities Space Research Association, Columbia, MD 21046, USA
} 


\section{Introduction}

Magnetars have fascinated high-energy astrophysicists for four decades, propelled by the first observation of a giant flare on 5th March 1979 from the soft gamma repeater SGR 0525-66 [34]. They are highly-magnetized ( $B \gtrsim 10^{14}$ Gauss) neutron stars that have historically been divided into two observational groups: Soft-Gamma Repeaters (SGRs) and Anomalous X-ray Pulsars (AXPs). Their extreme fields are inferred directly from their timing properties presuming that their rapid rotational spin down is due to magnetic dipole torques (e.g. see [29]). Such a class of neutron stars with superstrong fields was postulated as a model for SGRs by [15], and for AXPs by [44]. For reviews of magnetar science, see [36, 46,28]. Since their magnetic fields exceed the quantum critical value of $B_{\mathrm{cr}}=m_{e}^{2} c^{3} /(e \hbar) \approx 4.41 \times 10^{13}$ Gauss (the Schwinger value where the cyclotron energy $\hbar\left(e B / m_{e} c\right)$ of the electron equals its rest mass energy $\left.m_{e} c^{2}\right)$ the treatment of exotic processes in QED is mandated. This physics regime is not presently accessible by terrestrial experimental facilities, rendering cosmic magnetars an important testbed for physics theory.

SGRs, over a dozen in number, are transients that exhibit repeated soft gamma-ray bursts of subsecond duration in the $10^{38} \mathrm{erg} / \mathrm{sec}<L<10^{42} \mathrm{erg} / \mathrm{sec}$ range, though three of them have exhibited giant super-second flares of energies exceeding $10^{45}$ ergs (e.g. see [26] for SGR 1900+14, and [40] for SGR 1806-20), flares that could possibly be accompanied by gravitational wave signals detectable by aLIGO. They also exhibit quiescent emission with periods $P$ in the range 2-12 sec. (e.g. see [29], for SGR 1806-20). The AXPs are a group of around a dozen pulsating, steady, bright X-ray sources with similar periods. Their quiescent signals are mostly thermal with steep power-law tails (e.g. [41, 47]), and flat, hard X-ray tails (e.g., [31, 30, 13, 14]) that are the subject of this paper. AXPs possess persistent luminosities $L_{X} \sim 10^{35} \mathrm{erg} \mathrm{s}^{-1}$; as with the SGRs, these $L_{X}$ values far exceed their rotational power, so they are possibly fueled by their internal magnetic energy. Observations of outburst activity in AXP 1E 2259+586 [18], in AXP 1E1841-045 [33], and in others suggest that anomalous X-ray pulsars are indeed very similar to SGRs. This "unification paradigm" has garnered widespread support within the magnetar community over the last decade. The observational status quo of magnetars is summarized in the McGill Magnetar Catalog [39]. ${ }^{1}$

The discovery by INTEGRAL and RXTE of hard, non-thermal pulsed spectral tails in AXPs $[31,30,13,14]$ added to the magnetar mystique by signalling the existence of a sustained magnetospheric component to their radiative resumé. These luminous tails are extremely hard, typically extending up to $150-200 \mathrm{keV}$, but with a turnover below around $500 \mathrm{keV}$ implied by constraining pre-2000 COMPTEL upper limits (see Fig. 1). Similar persistent emission tails are seen in SGRs (e.g. [20] for SGR 1900+14). Fermi-GBM has also observed these tails [8], providing the sensitivity to better measure the flux above $100 \mathrm{keV}$ (L. Kuiper, private communication). The pulse profile and spectrum for AXP 1E 1841-045 are exhibited in Fig. 1, with the latter suggesting possible evidence for a turnover at around $150 \mathrm{keV}$. It is notable that this tail emission component, now seen in 9 magnetars, is not detected by the Fermi-LAT [1,32]. It is this hard X-ray component that is the subject of an ongoing investigation by our team, some details of which we present here as we progress along the labyrinthine path for demystifying the magnetospheres of magnetars.

\footnotetext{
${ }^{1}$ An on-line version is at http://www.physics.mcgill.ca/pulsar/magnetar/main.html; for a compendium of burst observational papers, see also https://staff.fnwi.uva.nl/a.l.watts/magnetar/mb.html.
} 

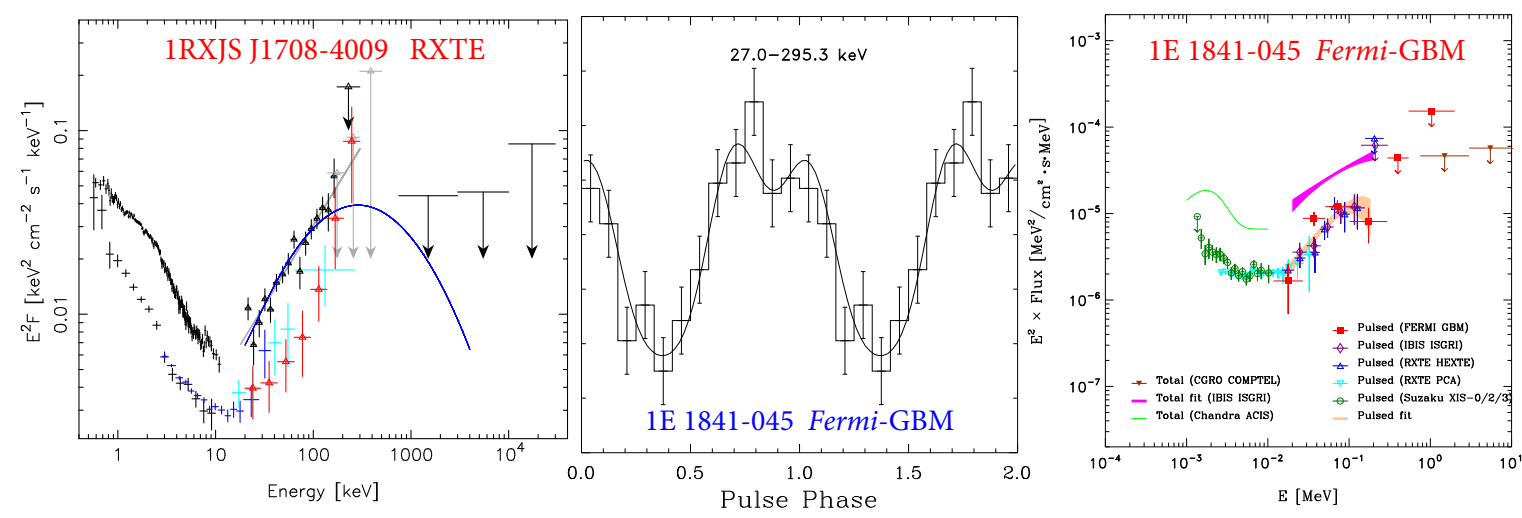

Figure 1: Left panel: The $v F_{v}$ X-ray spectrum of the AXP 1RXJS J1708-4009, with XMM data below $10 \mathrm{keV}$, and RXTE-PCA/HEXTE data above $10 \mathrm{keV}$ (red=pulsed) defining the hard X-ray tail. The noncontemporaneous COMPTEL upper limits above $1 \mathrm{MeV}$ are also shown, as is an empirical spectral fit (blue) - see Fig. 4 of [14]. Middle panel: Pulse profile of AXP 1E 1841-045 using Fermi-GBM data - Fig. 18 of [8]. The smooth line shows the template profile observed by INTEGRAL IBIS-ISGRI, 10-Mar-2003 to 30-Sep2009, at 50-150 keV. Right panel: The spectrum of quasi-thermal ( $\lesssim 10 \mathrm{keV}$; surface) and tail ( $\gtrsim 10 \mathrm{keV}$; magnetosphere) quiescent emission from 1E 1841-045, with pulsed emission represented by points: red is Fermi-GBM (maybe with a break at $\sim 150 \mathrm{keV}$ ), and other points constitute Chandra, RXTE, Suzaku and INTEGRAL data. COMPTEL upper limits above $1 \mathrm{MeV}$ are also shown. See Fig. 25 in [8].

\section{Hard X-ray Tail Modeling Essentials}

Opacity: The most efficient means for producing the hard tails in the $10-200 \mathrm{keV}$ range is via resonant inverse Compton scattering (RICS) by energetic electrons. This is the leading scenario for the production of this signal, where relativistic electrons energized in the inner magnetosphere upscatter surface thermal $\mathrm{X}$ rays of $k T_{s} \sim 0.5 \mathrm{keV}([5,17,38]$, and later papers). In this picture, Thomson optically thin conditions exist. To discern this, let $\mathscr{E}_{e} \gtrsim L_{\gamma} /\left(4 \pi R^{2} c\right)$ be the representative kinetic energy density in radiating electrons/pairs of mean Lorentz factor $\left\langle\gamma_{e}\right\rangle \sim 10-100$. Since the electron number density is $n_{e} \sim \mathscr{E}_{e} /\left(\left\langle\gamma_{e}\right\rangle m_{e} c^{2}\right)$, one quickly arrives at the non-magnetic Thomson optical depth $\tau_{\mathrm{T}}=n_{e} \sigma_{\mathrm{T}} R \gtrsim L_{\gamma} \sigma_{\mathrm{T}} /\left(4 \pi R m_{e} c^{3}\left\langle\gamma_{e}\right\rangle\right)$. For $R \sim 10^{6} \mathrm{~cm}$ and the observed persistent hard X-ray luminosities $L_{X} \sim 10^{35} \mathrm{erg} / \mathrm{sec}$, this yields $\tau_{\mathrm{T}} \sim 10^{-4}-10^{-3}$, i.e., populations of density $n_{e} \gtrsim 10^{15}-10^{16} \mathrm{~cm}^{-3}$ that exceed the Goldreich-Julian value $n_{\mathrm{GJ}}=|\nabla \cdot \mathbf{E}| / 4 \pi e \sim B /($ Pce $)$ by several orders of magnitude [5]. This opacity estimate increases by 2-3 orders of magnitude for scattering at the cyclotron resonance, which naturally arises in the magnetosphere and defines the high efficiency and the central character of the RICS model for magnetar hard tail production.

Our group has developed a refined upscattering model for this $>10 \mathrm{keV}$ emission over recent years. The nominal geometry for this picture is depicted in Fig. 2 (left), with relativistic electrons traveling along the (red) field lines $\left(\mathbf{p}_{e} \| \mathbf{B}\right)$ in these slow rotators. At a scattering point somewhere on a closed field line in the magnetosphere, a relativistic electron energized perhaps by currentdriven twisted fields possessing toroidal components $[45,9,11]$ collides with a thermal X-ray (of energy $\varepsilon_{s} m_{e} c^{2}$ ) emanating from the stellar surface within a cone of collimation. If the kinematic conditions are just right, namely $\gamma_{e} \varepsilon_{s}\left(1-\cos \theta_{\mathrm{kB}}\right)=B$, the scattering samples a strong resonance at the cyclotron frequency. Here $\theta_{\mathrm{kB}}$ is the angle between the photon momentum and $\mathbf{B}$, and hereafter magnetic field strengths $B$ will be expressed in units of $B_{\mathrm{cr}} \approx 4.41 \times 10^{13}$ Gauss, the 
quantum critical value. The full QED cross section of Gonthier et al. [19] for $B=3$ is depicted in the central panel of Fig. 2, and the prominent resonance with $\sigma / \sigma_{\mathrm{T}} \sim 10^{2}-10^{3}$ is obvious. The reader can consult $[10,23]$ for the simpler special case of magnetic Thomson scattering.
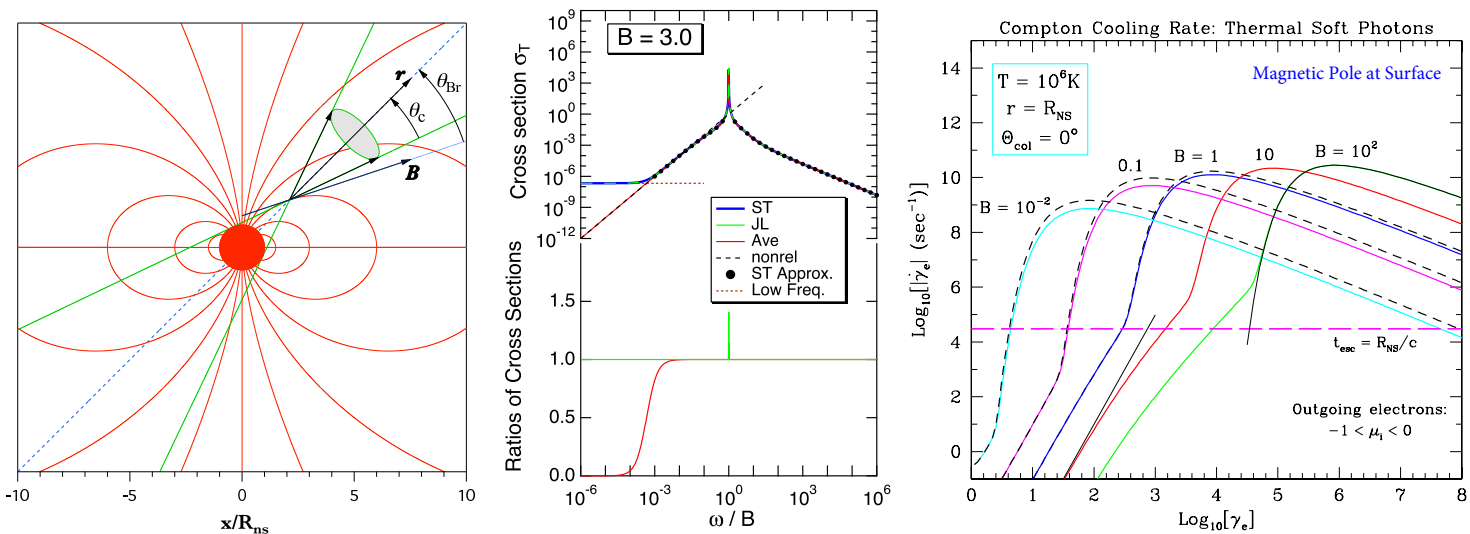

Figure 2: Left panel: The magnetospheric geometry for inverse Compton scatterings at arbitrary altitudes and colatitudes in the magnetosphere: see [7]. The green "cone" represents the collimated soft photons from the surface for an interaction point located at $r / R_{\mathrm{NS}}=3$ and a colatitude of $45^{\circ}$. The spatial scale is linear, in units of $R_{\mathrm{NS}}$. Center panel: Total QED magnetic Compton cross sections (in units of $\sigma_{\mathrm{T}}$ ) in the ERF [19], averaged over polarization (upper part), in the case of photons moving along $\mathbf{B}$. The spin-dependent Johnson \& Lippman (JL; green solid), the Sokolov \& Ternov (ST; blue solid), and the spin-averaged (red solid) cross sections are displayed for $B=3$ (in units of $B_{\mathrm{cr}}$ ), and in the lower part the ratios of the JL (green) and average (red) cross sections to that of ST cross section are displayed. Only ST is accurate in the cyclotron resonance $\omega \approx B$. Right panel: Resonant Compton cooling rates [7] for relativistic electrons colliding with thermal $\mathrm{X}$ rays $\left(T=10^{6} \mathrm{~K}\right)$ in different fields. Solid curves are for the correct ST choice for electron spinors, dashed are for the JL choice. The approximate Lorentz factor $\gamma_{e}$ of the rapid rise or "wall" correlates with $B$ because of the approximate resonant scattering condition $\gamma_{e} k T / m_{e} c^{2} \sim B$. The horizontal dashed line denotes the light escape scale $1 / t_{\mathrm{esc}}=c / R_{\mathrm{NS}}$ corresponding to a stellar radius.

QED Scattering Physics: For precision computations of the RICS process in magnetars, accurate full QED formulations of magnetic Compton scattering are requisite, including the kinematics of electron recoil and Klein-Nishina reductions. State-of-the-art analytics and computations of the polarization-dependent, magnetic Compton differential cross section $d \sigma_{\mathrm{T}} / d \Omega$ in the electron rest frame (ERF) have been delivered in [19], and are used in our various modeling papers. These results incorporate the formally appropriate Sokolov \& Ternov (ST) [42] spinor formalism, in which the wavefunction solutions to the magnetic Dirac equation diagonalize the spin operator $\mu_{z}$ parallel to the field. These wavefunctions have become a preferred choice in strong-field QED calculations over the last two decades (as opposed to the older Johnson \& Lippmann (JL) [27] eigenstates), since they capture important symmetries. The ST wavefunctions are symmetric between $e^{-}$and $e^{+}$states [24, 35]. Also, [21, 6] established that under Lorentz boosts along $\mathbf{B}$, ST states transform unmixed and yield cyclotron rates that are modified simply via boost Lorentz factors; in contrast, JL states do not. The inclusion of a cyclotron decay width $\Gamma$ for the intermediate virtual electron state describes its lifetime, and is essential, rendering the cross section finite at the cyclotron resonances. This standard Breit-Wigner protocol must employ spin-dependent widths [22, 21, 6], and the JL states are not appropriate for correctly implementing such, but the Sokolov \& Ternov eigen- 
states are. The developments in [19] focused on the singular case of incident photons propagating along $\mathbf{B}$, i.e. $\theta_{\mathrm{kB}}=0$, a suitable ERF specialization for the RICS modeling where $\gamma_{e} \gg 1$ introduces relativistic aberration. In particular, [19] clearly demonstrated the improved precision of the cross section in the resonance when employing ST eigenstates (confirmed by [37]), revealing the discrepancies incurred when employing JL states: for an example, see the middle panel of Fig. 2.

Cooling Rates: The focus of our magnetar X-ray tail work first centered on computations of electron cooling rates due to resonant inverse Compton (IC) scattering [7] of soft X-rays from entire, isothermal neutron star surfaces. These rates were derived using ST spinor formalism QED cross sections for scattering, and clearly demonstrated the inaccuracies inherent in magnetic Thomson evaluations. The cooling calculations were performed in flat spacetime dipole magnetospheres to identify the essential features. Sample cooling rates $\dot{\gamma}_{e}$ and their dependence on $\gamma_{e}$ and field strength $B_{p}$ at the magnetic polar surface are illustrated in Fig. 2 (right panel). The forms are essentially inverted images of Planck spectra governed by the resonant kinematic criterion $\gamma_{e} \varepsilon_{i} \sim B$ for seed $\mathrm{X}$ rays of energy $\varepsilon_{i} m_{e} c^{2} \sim 3 k T \approx 0.25 \mathrm{keV}$ (i.e., $\varepsilon_{i} \sim 5 \times 10^{-4}$ for $T=10^{6} \mathrm{~K}$ ). The rapid rise in $\dot{\gamma}_{e}$ when $\gamma_{e}$ approaches $B / \varepsilon_{i}$ can limit putative processes that energize the relativistic electrons in the first place. Such radiation reaction would then control the maximum $\gamma_{e}$ of electrons, yielding values dependent on the magnetospheric locale. Fig. 2 indicates that generally, resonant scattering will impose a limit $\gamma_{e}<10^{5}$ in polar fields $B_{p} \sim 10^{2}$. Moreover, in the lower local fields of $B \lesssim 0.1$ encountered at higher altitudes and equatorial colatitudes along closed field lines, RICS will de-energize fast electrons down to Lorentz factors $\gamma_{e}$ of the order of 5-20 on lengthscales of $\ell \sim 1-10 \mathrm{~cm}$. These low $\gamma_{e}$ in turn ascribe low values to the maximum photon energy $\sim \gamma_{e}^{2} \varepsilon_{i}$ in the IC spectrum, values around $150 \mathrm{keV}$ for $\gamma_{e} \sim 10$. Accordingly, the observed maximum energies of the hard tails in the $<300 \mathrm{keV}$ range might be indicative of the curtailment of electron energization by radiation reaction, although it could be caused by spectral attenuation: see below.

Spectra: The next chapter in our studies produced an array of spectral results from uncooled electrons in dipolar field geometry. Representative spectra for monoenergetic electrons are offered in Wadiasingh et al. [48], with Fig 3 showing a $B_{p}=10$ case for two Lorentz factors $\gamma_{e}=10,100$. The curves therein (normalized to roughly match the data) correspond to a fixed viewing angle $\theta_{v}=$ $30^{\circ}$ with respect to the instantaneous magnetic dipole axis $\hat{\boldsymbol{\mu}}$ (note that $\theta_{v}$ varies sinusoidally with phase as the star rotates). Therefore these spectra would be sampled at a particular rotational phase of the magnetar during its spin period. This illustration displays what an observer would see from a complete closed magnetic field line (footpoint to equator to footpoint) if the line of sight is coplanar with the field loop $\left(\phi_{0}=0^{\circ}\right)$. This is a rare case that enables potentially detectable emission out to $\sim \gamma_{e}^{2} \varepsilon_{i} m_{e} c^{2} \sim 10 \mathrm{MeV}$ when $\gamma_{e}=100$, for scattering in the Thomson limit $\left(\gamma_{e} \varepsilon_{i} \ll 1\right)$. For viewing angles oblique to this plane containing the loop (i.e., $\phi_{0}>0$ ), the spectra are much softer [48]. Observe the mismatch between the model spectral slopes and the data [14]. This is not of concern because the computations were for electrons with no energy losses moving along a single loop, and in the absence of attenuation processes such as pair creation and photon splitting. The spectra are parameterized by $r_{\max } R_{\mathrm{NS}}$, the equatorial altitude of each magnetic loop, with $r_{\max }$ sampling a logarithmic scale. The prominent cusps at the highest energies are due to strong Doppler boosting of upscattered emission when the line of sight is tangent to a field line, i.e. parallel to $\mathbf{p}_{e}$. 

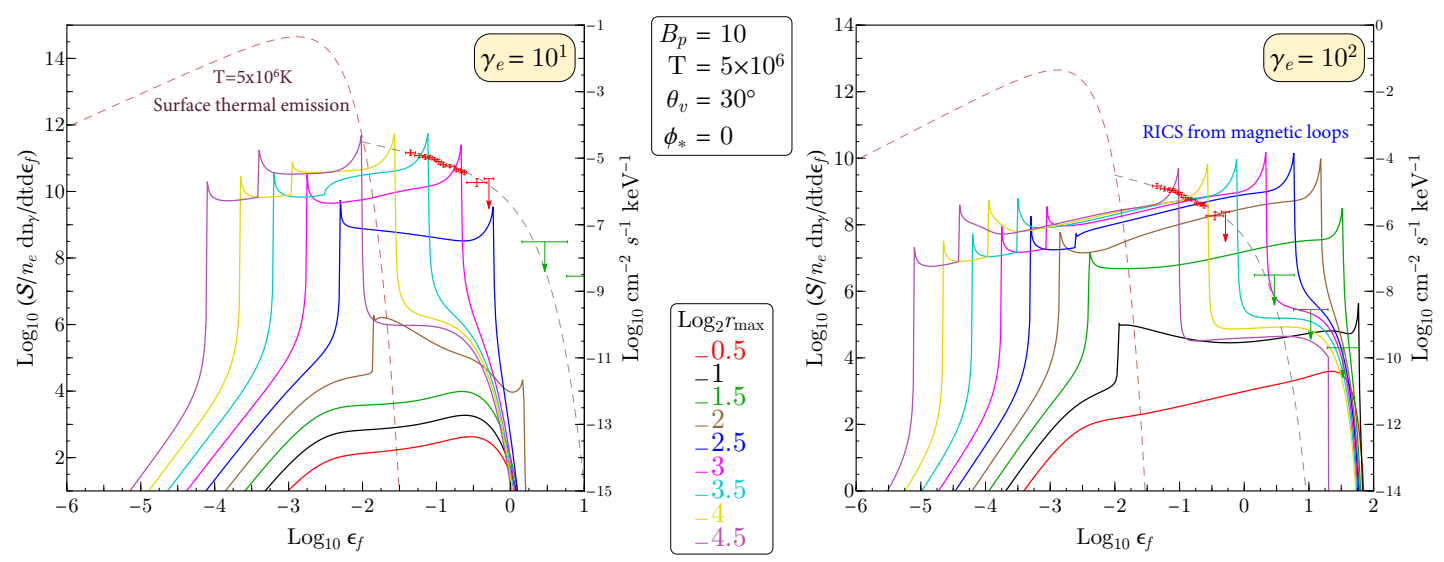

Figure 3: Unnormalized RICS upscattering $F_{v} / v$ spectra for meridional field loops, coplanar with the viewer's direction, depicting nine choices of the maximum loop altitude parameter $r_{\max }=\left\{2^{0.5}, \ldots, 2^{4.5}\right\}$ (in units of $R_{\mathrm{NS}}$ ). The two panels are for different Lorentz factors $\gamma_{e}$. The superposition of these curves gives an indication of spectra that might result from toroidal volumes. Fig. 10 of Wadiasingh et al. [48]. Star parameters are the surface polar field $B_{p}=10$ and the uniform surface temperature $T=5 \times 10^{6} \mathrm{~K}$. Spectra are realized for a viewing angle of $\theta_{v}=30^{\circ}$ to the magnetic axis (a particular pulse phase). Observational data points [14] for AXP 4U 0142+61 are overlaid (red), plus COMPTEL upper limits, along with a schematic $\varepsilon_{f}^{-1 / 2}$ power-law with a $250 \mathrm{keV}$ exponential cutoff (gray dashed curve). The Planck spectrum $(k T=0.41 \mathrm{keV})$ approximating the soft $\mathrm{X}$-ray data for this magnetar is indicated by the brown dashed curve.

The broader ensemble of spectra in [48] indicates that AXP/SGR hard tails with cutoffs below $500 \mathrm{keV}$ are best generated when the observer viewing angles to the magnetic field are typically greater than $\sim 3^{\circ}$. Such a simple conclusion is based in the kinematics of resonant upscattering. The produced photon energy $\varepsilon_{f}$ (in units of $m_{e} c^{2}$, and often scaling as $\propto \gamma_{e}^{2} \varepsilon_{i}$ ) and the scattering angle $\theta_{f}$ with respect to the local field $\mathbf{B}$ are strongly correlated via the Doppler boosting condition $\gamma_{e} \varepsilon_{f}\left(1-\cos \theta_{f}\right) \sim B$. Super-MeV photons are then only observed in magnetars when $\theta_{f}$ is small (typically a few degrees). Since, for a particular observer perspective that is not along the magnetic dipolar axis, the local field is seldom tangent to the line of sight, the emergent spectra in static magnetospheres then indicate softer emission below around $1 \mathrm{MeV}$ when $\gamma_{e} \lesssim 20$. These are not too disparate with data for the hard X-ray tail cutoffs, as is apparent in the comparison between the $\gamma_{e}=10$ and $\gamma_{e}=100$ cases exhibited in Fig. 3. Moreover, the cooling rates depicted at the right in Fig. 2 suggest that rampant resonant Compton cooling at magnetar field strengths may limit electron Lorentz factors to around $\gamma_{e} \sim 10$, indicating approximate spectral consistency with the observations. Note that for viewing angles approximately above the pole, resonant scattering can proceed at much higher altitudes where the field is lower (especially if $B_{p} \lesssim 1$ ), reducing the cooling and increasing the possibly Lorentz factors to $\gamma_{e}>10^{3}$. The associated Doppler boosting should precipitate much harder spectra, perhaps consistent with those seen in high-field pulsars.

The spectra in Fig. 9 of [48] are strongly polarized near the maximum resonant scattering energies (see also [5]) due to the intrinsic dependence of the Compton cross section on the polarization of the scattered photon [19]: the $\perp$ state always exceeds the $\|$ state. Here, as always, we adopt a standard linear polarization convention: $\|$ (O-mode) refers to the state with the photon's electric field vector parallel to the plane containing $\mathbf{B}$ and the photon's momentum vector, while $\perp$ (X-mode) denotes the photon's electric field vector being normal to this plane. This polarimetric 

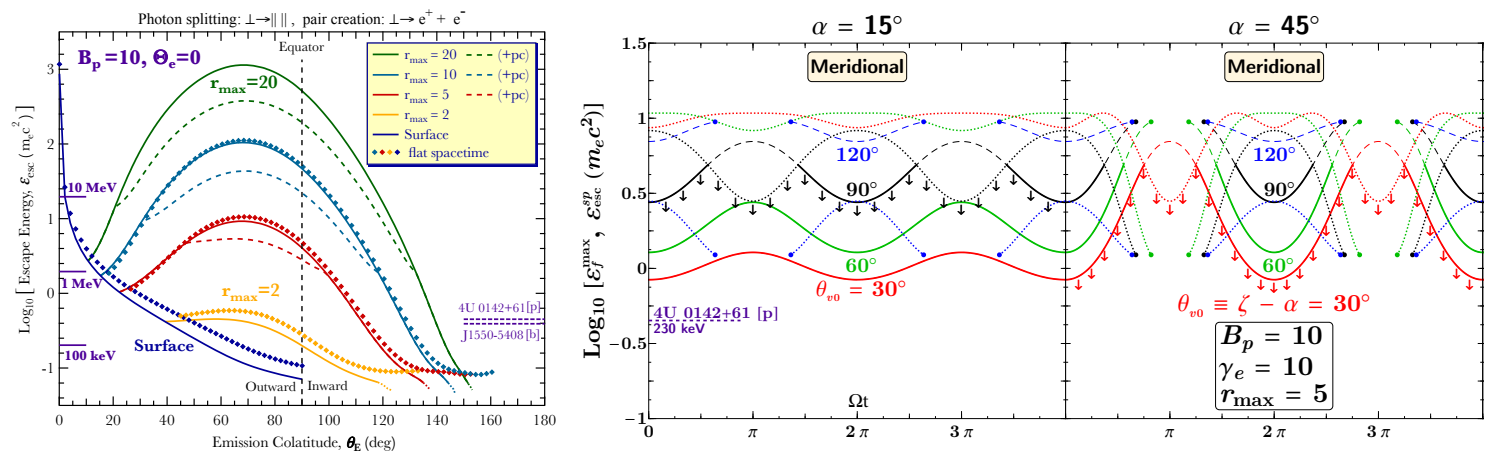

Figure 4: Left panel: Escape energies for photon splitting (mode $\perp \rightarrow\|\|$, solid curves) and also when adding pair creation of the $\perp$ state to the total opacity ( + pc, dashed), for surface polar fields $B_{p}=10$. All determinations are for general relativistic propagation, except for those denoted as being for flat spacetime, namely the traces of diamonds for the surface and $r_{\max }=2,5,10$ examples. A meridional loop specialization is adopted, with photon emission initially parallel to the local magnetic field line $\left(\Theta_{\mathrm{e}}=0\right)$, for maximum loop altitudes $r_{\max }=2,5,10,20$ in units of $R_{\mathrm{NS}}$. The surface emission curves are truncated at the equator. The maximum observed energies for persistent [p] hard tail emission from $4 \mathrm{U} 0142+61(230 \mathrm{keV})$ and burst emission [b] from SGR J1551-5408 are marked. Right two panels: Flat spacetime photon splitting escape energy $\varepsilon_{\text {esc }}^{s p}$ (dotted curves) for $\perp \rightarrow\|\| \|$ and resonant Compton maximum cutoff energy $\varepsilon_{f}^{\max }$ (solid and long-dashed) as functions of spin phase $\Omega t$, for oblique rotators with $\alpha \equiv \arccos (\hat{\boldsymbol{\Omega}} \cdot \hat{\boldsymbol{\mu}})=\left\{15^{\circ}, 45^{\circ}\right\}$. Curves are depicted for several choices of the particular observer viewing angle $\theta_{v 0}=\zeta-\alpha$ to $\hat{\mu}$ at phase zero $(\cos \Omega t=1)$, as labeled and color-coded. The $\varepsilon_{f}^{\max }$ emission energies are depicted as solid curves if $\varepsilon_{f}^{\max }<\varepsilon_{\text {esc }}^{s p}$, and as long-dashed loci otherwise, when $\perp$ photons are attenuated at energies below $\varepsilon_{f}^{\max }$. Curves terminate at dots marking field line footpoint emission locales. Downward arrows mark two sample profiles for effective maximum energies observed in the $\perp$ state, signifying $\min \left\{\varepsilon_{f}^{\max }, \varepsilon_{\text {esc }}^{s p}\right\}$. From [25].

signature defines a strong motivation for developing future hard X-ray polarimeters.

Spectral Attenuation: The RICS emission does not necessarily survive to emerge to infinity, particularly at energies above $50 \mathrm{keV}$. The inner magnetosphere is potentially opaque to the escape of such energetic photons due to two QED processes that are efficacious in strong magnetic fields: single-photon pair creation $\gamma \rightarrow e^{+} e^{-}$and photon splitting $\gamma \rightarrow \gamma \gamma$. The mechanism $\gamma \rightarrow e^{ \pm}$is active above the $2 m_{e} c^{2} \approx 1.02 \mathrm{MeV}$ threshold [16, 12, 4], is the absorptive part of the birefringent dispersion of the magnetized QED vacuum, and is central to the foundation of early pulsar models. Story \& Baring [43] computed $\gamma$-ray opacity for pulsars due to pair creation, determining that it limits emission to energies below $\sim 10-30 \mathrm{MeV}$ along polar field lines when $B_{p} \gtrsim 10$. This would explain why the Fermi-LAT instrument has not detected magnetars [1]. This bound moves to lower energies for equatorial interaction zones with increased field line curvature [25], since the attenuation rate is a strongly increasing function of the photon angle $\theta_{\mathrm{kB}}$ to $\mathbf{B}$. Hu et al. [25] presented a comprehensive analysis of pair creation and photon splitting opacity in dipolar magnetar magnetospheres, in both flat and curved spacetime.

Splitting is a higher-order QED process, of the order of $\alpha_{\mathrm{f}}^{2}=\left(e^{2} / \hbar c\right)^{2}$ smaller in rate than pair creation $\gamma \rightarrow e^{ \pm}$. It is extremely polarization-dependent so that the interplay between scattering and splitting is profound. The splitting modes $\perp \rightarrow\|\|,\|\rightarrow \perp\|$ and $\perp \rightarrow \perp \perp$ are the only ones permitted by CP invariance in the limit of zero dispersion [3, 4]. Adler's [2] selection rules for $\gamma \rightarrow \gamma \gamma$ argue that only the $\perp \rightarrow\|\|$ mode of splitting operates in the birefringent, magnetized QED vacuum. 
Since photon splitting has no threshold imposed by mass creation, it can proceed at energies below $1 \mathrm{MeV}$, and is influential in magnetars down to energies of $50 \mathrm{keV}$ or so [4]. Below pair threshold, the splitting rate scales roughly with energy as $\varepsilon^{5}$ [2,3]. The trajectory integral analysis of [25] provided upper bounds of a few $\mathrm{MeV}$ or less to the visible energies for magnetars for locales proximate to the stellar surface. This is illustrated in the left panel of Fig. 4, which depicts the maximum energy $\varepsilon_{\text {esc }}$ of photons that can escape the magnetosphere from select closed field lines and also the stellar surface. These escape energies are depicted therein as functions of emission colatitude for the $\perp$ polarization state only, with solid curves for splitting, and dashed curves isolating pair creation when it lowers the escape energy at quasi-equatorial colatitudes. Photons emitted in regions within field loops of maximum altitudes $r_{\max } \sim 2-5$ would be attenuated by photon splitting at energies below around $250 \mathrm{keV}$, the highest detected in hard X-ray tails. The zones of exclusion are asymmetric between upward/downward emission hemispheres.

To combine the information on RICS spectroscopy and attenuation escape energies, [25] outlined the polarization-dependent effective maximum energies $\varepsilon_{f}^{\max }$ for resonant upscattering subject to attenuation by photon splitting $\left(\varepsilon_{\mathrm{esc}}^{s p}\right)$. A subset of the results from Fig. 8 of that work appears in Fig. 4, wherein the pulse phase dependence of these energies is depicted. These were computed for a $B_{p}=10$ magnetar in flat spacetime and a magnetic loop with $r_{\max }=5$. The two panels contrast an almost aligned rotator $\left(\alpha=15^{\circ}\right)$ with an oblique one, illustrating the increase in modulation with $\alpha$, which is the angle between the spin axis $\boldsymbol{\Omega}$ and the magnetic dipole axis $\mu$. The meridional case is for when the electrons traverse magnetic field loops that possess locales with tangents pointing toward an observer, corresponding to intense and hard radiation signals at particular phases due to strong beaming. Thus $\varepsilon_{f}^{\max }$ exceeds the observed maxima for the hard tails, and photon splitting helps attenuate, but generally only above around $2 \mathrm{MeV}$. The contrasting case of anti-meridional motions, where the Doppler beaming is away from the observer, renders $\varepsilon_{f}^{\max }$ below $100 \mathrm{keV}$. Then, photon motions are generally inward, and the attenuation by splitting is prolific, suppressing emission above $100 \mathrm{keV}$ - see Fig. 8 of [25]. The upshot is that sensitive phase-dependent spectroscopy+polarimetry above $100 \mathrm{keV}$ will afford tight constraints on the RICS model parameters such as $\gamma_{e}$ and the set of field lines that are actively generating the emission. Such diagnostics may be enabled by the planned medium $\gamma$-ray energy telescope AMEGO. ${ }^{2}$

\section{Volume-Integrated Spectroscopy and Pulse Profiles}

The select spectra in Fig. 3 from single magnetic field loops do not represent an ensemble summation over a bundle of field lines. The extension to integrations of RICS signals over magnetospheric volumes is detailed in a new paper Wadiasingh et al. (2021, ApJ in prep. [49]), wherein spectra are obtained using a versatile $\mathrm{C}++$ code, again with fixed electron $\gamma_{e}$, and in flat spacetime. One of the main results of this new installment of our program is that the particular integration over a wide array of field lines determines the spectral index below the RICS cutoff or effective maximum energy. The principal effect of integrating over toroidal field volumes defined by ranges of $r_{\max }$ and magnetic longitudes, as opposed to individual field lines or toroidal surfaces with one value of $r_{\max }$, is to populate lower frequencies more, so that the spectra steepen on average. An

\footnotetext{
${ }^{2}$ AMEGO web page: https://asd.gsfc.nasa.gov/amego/index.html
} 
illustrative selection of volume-integrated spectra is exhibited in the left panel of Fig. 5, where results for five ranges of $r_{\max }$ are depicted. Attenuation by photon splitting and pair creation is omitted from this example, though it is treated at length in [49]. Corresponding to emission from a hollow toroidal volume, these spectra are steeper than those in Fig. 3 (observe the $v F_{v}$ representation). They therefore match the $4 \mathrm{U} 0142+61$ INTEGRAL/RXTE data much more closely when $\gamma_{e}=10$, particularly for $2 \leq r_{\max } \leq 2^{4.5 \rightarrow 5.5}$, something that doesn't arise when $\gamma_{e} \gtrsim 100$ and the COMPTEL upper bounds are violated [49]. Accordingly, the phase-averaged spectra serve as a diagnostic on both the mean Lorentz factor $\gamma_{e}$ and the thickness of the active toroidal volume, at least modulo the assumed dipolar field geometry. Observe the appearance of prominent low frequency bumps when high altitudes with $r_{\max } \sim 2^{6}-2^{7}$ are incorporated, precipitated by the preponderance of low fields; while obviously interesting, these may be precluded by the $<10 \mathrm{keV}$ spectral shape. Fig. 5 also indicates a high degree of polarization of the radiation from the scattering, a signature that can be leveraged by future hard X-ray polarimeters.
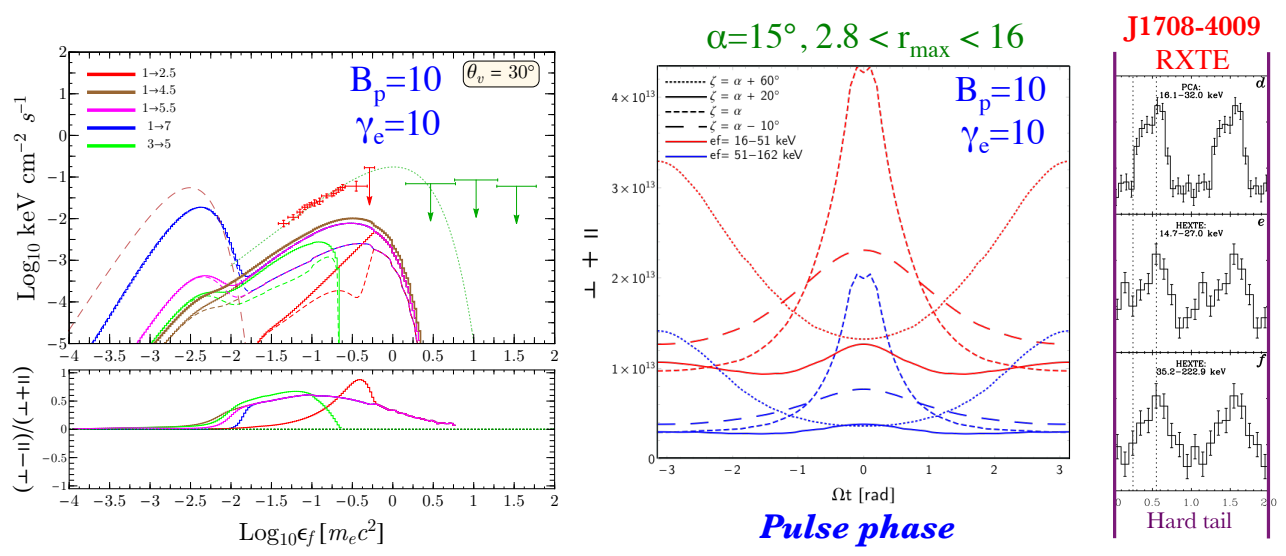

Figure 5: Left panel: Polarized inverse Compton spectra ([49]; note the $v F_{v}$ representation) for $\gamma_{e}=10$, from integrations over toroidal volumes of field loops. These volumes comprise azimuthally-integrated toroidal surfaces specified by $r_{\max }=2^{\kappa}$, where $\kappa$ spans the ranges in the legend, each depicted for two polarizations: $\perp$ (solid) and $\|$ (dashed); the bottom panel displays the polarization degree. Center panel: Pulse profiles for a toroidal volume integrations $r_{\max }=2^{1.5} \rightarrow 2^{4}$, generated by a range of $\theta_{v}$ values sampled by a rotating magnetar. Red profiles are for $16-51 \mathrm{keV}$, and blue are for $51-162 \mathrm{keV}$, being obtained for different observer viewing angles $\zeta$ to the rotation axis. Results are for $B_{p}=10$ and rotator magnetic inclination $\alpha=15^{\circ}$, and electron Lorentz factor $\gamma_{e}=10$. Right panel: Observed RXTE PCA+HEXTE profiles for different hard X-ray bands for RXS J1708-4009 adapted from Fig. 1 of [30].

Pulse Profiles: A new element of our program is the generation of pulsation profiles for comparison with observations. Fluxes in any given waveband can routinely be obtained for a rotating star in flat spacetimes. The inclination angle $\alpha$ between the magnetic dipole $\boldsymbol{\mu}$ and spin axis $\boldsymbol{\Omega}$ vectors specifies the rotator geometry. The observer viewing geometry is prescribed via the aspect angle $\zeta$ between the line of sight $\mathbf{O}$ and spin axis $\boldsymbol{\Omega}$ vectors. Angles $\alpha$ and $\zeta$ are standard stellar parameters for pulsar studies. The instantaneous viewing angle $\theta_{v}$ varies in a sinusoidal fashion as the magnetar rotates [48], with its average corresponding to $\alpha$, and its amplitude being $|\zeta-\alpha|$. Spectra analogous to those in Fig. 3 are obtained for an array of $\theta_{v}$, integrated over set frequency ranges and presented as intensity "sky maps" in [49]; these maps have pulse phase 
$\Omega t$ on the abscissa, and $\zeta$ as the ordinate. Horizontal cuts through these sky maps produce pulse profiles, and we exhibit such in the center panel of Fig. 5 for a rotator inclination $\alpha=15^{\circ}$. These profiles, which in general depend on the thickness of the toroidal emission volume, clearly display first and second-order Fourier components that result from the azimuthally-symmetric and hemispherically-symmetric volumes adopted in the integration. Real pulse profiles such as those for RXS J1708-4009 on the right of Fig. 5 display more harmonic structure, providing evidence for departures from these spatially-congruent specializations. A future stage of our program will consist of an exploration of these subtleties, using the pulse profile data to inform and constrain the azimuthal/longitudinal dimension of the radiating toroidal volume. Yet, with just the information presented in Fig. 5, it is evident that pulse profile comparison between model and data provides powerful diagnostics on stellar parameters $\alpha$ and $\zeta$, as well as the $r_{\max }$ range. Notably, pulse profiles with simple quasi-sinusoidal traces generally preclude values of $\alpha \gtrsim 40^{\circ}$ as they generate higher Fourier components [49] due to visibility of emission from both hemispheres.

\section{Conclusion}

The results surveyed here from our extensive program exploring resonant inverse Compton scattering models for magnetar hard X-ray tails display the evolution and depth of the analyses, the complexity of the modeling. The considerable sophistication of these undertakings will be further enhanced when the spectral and temporal emission information is combined with concurrent RICS cooling of the electrons. This serves as the next stage of this enterprise, which is well-positioned to afford observational diagnostics with extant pulse profile and spectral data from RXTE, INTEGRAL, NuSTAR and Fermi-GBM. Yet the polarimetric element of our studies is an attractive antecedent to a future era of hard X-ray polarimetry, when even more powerful probes of the mysteries of magnetar magnetospheres will be enabled. Foremost among these is the prospect of being able to experimentally demonstrate the verity of magnetic photon splitting and single-photon pair creation, and the intimately-connected birefringence of the magnetized quantum vacuum, heretofore untested theoretical predictions for high-field QED domains. Magnetars thus serve as a potent cosmic QED physics laboratory, and the model developments will foster this advance by disentangling source emission and geometry information from the signatures of strong-field QED physics.

Acknowledgements: M. G. B. acknowledges the generous support of the National Science Foundation through grant AST-1517550, and NASA's Fermi Guest Investigator Program through grant NNX16AR66G. Z. W. is supported by the NASA postdoctoral fellowship program.

\section{References}

[1] Abdo, A. A. et al. 2010, ApJ Supp. 187, 460

[2] Adler, S. L. 1971, Ann. Phys. 67, 599

[3] Baring, M. G. 2000, Phys. Rev. D, 62, 016003

[4] Baring, M. G. \& Harding, A. K. 2001, ApJ, 547, 929

[5] Baring, M. G. \& Harding, A. K. 2007, Astrophys. Spac. Sci., 308, 109

[6] Baring, M. G., Gonthier, P. L., \& Harding, A. K. 2005, ApJ, 630, 430

[7] Baring, M. G., Wadiasingh, Z., \& Gonthier, P. L. 2011, ApJ, 733, 61 
[8] ter Beek, F. 2012, Master's thesis, University of Amsterdam.

[9] Beloborodov, A. M. 2013, ApJ, 762, 13

[10] Canuto, V., Lodenquai, J., \& Ruderman, M. 1971, Phys. Rev. D, 3, 2303

[11] Chen, A. Y., \& Beloborodov, A. M. 2017, ApJ, 844, 133

[12] Daugherty, J. K. \& Harding, A. K. 1983, ApJ, 273, 761

[13] den Hartog, P. R., Kuiper, L., Hermsen, W., et al. 2008a, A\&A, 489, 245

[14] den Hartog, P. R., Kuiper, L. \& Hermsen, W. 2008b, A\&A, 489, 263

[15] Duncan, R. C. \& Thompson, C. 1992, ApJ Lett., 392, L9

[16] Erber, T. 1966, Rev. Mod. Phys., 38, 626

[17] Fernández, R. \& Thompson, C. 2007, ApJ, 660, 615

[18] Gavriil, F. P., Kaspi, V. M., \& Woods, P. M. 2004, ApJ, 607, 959

[19] Gonthier, P. L., Baring, M. G., Eiles, M. T., et al. 2014, Phys. Rev. D., 90, 043014

[20] Götz, D., Mereghetti, S., Tiengo, A., et al. 2006, A\&A, 449, L31

[21] Graziani, C. 1993, ApJ, 412, 351

[22] Harding, A. K., \& Daugherty, J. K. 1991, ApJ, 374, 687

[23] Herold, H. 1979, Phys. Rev. D, 19, 2868

[24] Herold, H., Ruder, H., \& Wunner, G. 1982, A\&A, 115, 90

[25] Hu, K., Baring, M. G., Wadiasingh, Z. \& Harding, A. K. 2019, MNRAS, 486, 3327

[26] Hurley, K., Kouveliotou, C., Woods, P., et al. 1999, ApJ Lett., 510, L107

[27] Johnson, M. H., \& Lippmann, B. A. 1949, Phys. Rev., 76, 828

[28] Kaspi, V. M., \& Beloborodov, A. M. 2017, Ann. Rev. Astron. Astrophys., 55, 261

[29] Kouveliotou, C., Dieters, S., Strohmayer, T., et al. 1998, Nature, 393, 235

[30] Kuiper, L., Hermsen, W., den Hartog, P. R. \& Collmar, W. 2006, ApJ, 645, 556

[31] Kuiper, L., Hermsen, W., \& Mendez, M. 2004, ApJ, 613, 1173

[32] Li, J., Rea, N., Torres, D. F. \& de Oña-Wilhelmi, E. 2017, ApJ, 835, 30

[33] Lin, L., Kouveliotou, C., Göğüş, E., et al. 2011, ApJ Lett., 740, L16

[34] Mazets, E. P., Golenetskii, S. V., Ilyinskii, V. N., et al. 1981, Astrophys. Spac. Sci., 80, 85

[35] Melrose, D. B., \& Parle, A. J. 1983, Aust. Journal Phys., 36, 755

[36] Mereghetti, S. 2008, A\&ARv, 15, 225

[37] Mushtukov, A. A., Nagirner, D. I., \& Poutanen, J. 2016, Phys. Rev. D, 93, 105003

[38] Nobili, L., Turolla, R., \& Zane, S 2008, MNRAS, 386, 1527

[39] Olausen, S. A. \& Kaspi, V. M. 2014, ApJ Supp., 212, 6

[40] Palmer D. M., et al., 2005, Nature, 434, 1107

[41] Perna, R., Heyl, J. S., Hernquist, L. E., et al. 2001, ApJ, 557, 18

[42] Sokolov, A. A. \& Ternov, I. M. 1968 Synchrotron Radiation, (Pergamon Press, Oxford)

[43] Story, S. A. \& Baring, M. G. 2014, ApJ, 790, 61

[44] Thompson, C. \& Duncan, R. C. 1996, ApJ, 473, 332

[45] Thompson, C., Lyutikov, M. \& Kulkarni, S. R. 2002, ApJ, 574, 332

[46] Turolla, R., Zane, S. \& Watts, A. L. 2015, RPPh, 78, 116901

[47] Viganò, D., Rea, N., Pons, J. A., et al. 2013, MNRAS, 434, 123

[48] Wadiasingh, Z., Baring, M. G., Gonthier, P. L. \& Harding, A. K. 2018, ApJ, 854, 95

[49] Wadiasingh, Z., Baring, M. G., Harding, A. K., et al. 2021, ApJ, in preparation. 